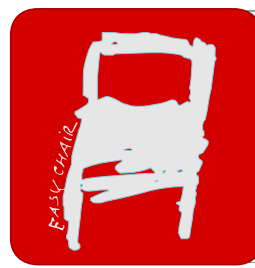

EPiC Series in Health Sciences

Volume 2, 2018, Pages 134-138

CAOS 2018. The 18th Annual Meeting of the International Society for Computer Assisted Orthopaedic Surgery

\title{
An Exploration of Augmented Reality in Computer Assisted Orthopedic Surgery
}

\author{
He Liu ${ }^{1 *}$, Edouard Auvinet ${ }^{2}$, Joshua Giles ${ }^{1,3}$ and Ferdinando Rodriguez y \\ Baena $^{1 \dagger}$ \\ ${ }^{1}$ Mechatronics in Medicine Laboratory, Imperial College, London, U.K. \\ ${ }^{2}$ MSk Lab, Imperial College, London, U.K. \\ ${ }^{3}$ Department of Mechanical Engineering, University of Victoria, Victoria, Canada \\ he.liu15@imperial.ac.uk, f.rodriguez@imperial.ac.uk
}

\begin{abstract}
Computer Aided Surgery (CAS) is helpful, but it clutters an already overcrowded operating theatre, and tends to disrupt the workflow of conventional surgery. In order to provide seamless computer assistance with improved immersion and a more natural surgical workflow, we propose an augmented-reality based navigation system for CAS. Here, we choose to focus on the proximal femoral anatomy, which we register to a plan by processing depth information of the surgical site captured by a commercial depth camera. Intra-operative three-dimensional surgical guidance is then provided to the surgeon through a commercial augmented reality headset, to drill a pilot hole in the femoral head, so that the user can perform the operation without additional physical guides. The user can interact intuitively with the system by simple gestures and voice commands, resulting in a more natural workflow. To assess the surgical accuracy of the proposed setup, 30 experiments of pilot hole drilling were performed on femur phantoms. The position and the orientation of the drilled guide holes were measured and compared with the preoperative plan, and the mean errors were within $2 \mathrm{~mm}$ and $2^{\circ}$, results which are in line with commercial computer assisted orthopedic systems today.
\end{abstract}

\section{Introduction}

Implantation accuracy is one of the most important considerations during joint replacement surfacing as it significantly impacts joint functional recovery and longevity (De Haan, Campbell, Su, \& De Smet, 2008) (Williams, Royle, \& Norton, 2009). Currently, bone resection and implant positioning are mainly achieved by the surgeon with the assistance of mechanical jigs, so the accuracy

${ }^{*}$ Created the first draft of this paper

$\dagger$ Principal investigator 
relies heavily on the surgeon's skills and experience, which can take significant time to develop. To improve surgeon accuracy, computer navigation is introduced into the procedure. Conventional computer navigation is displayed on two-dimensional monitors, which may lead to eye-hand coordination problems and dimension loss (Marescaux, Diana, \& Soler, 2013) (Abe, et al., 2013). In contrast, augmented reality (AR) overlays three-dimensional (3D) information in situ and does not block the real environment so that intuitive guidance can be provided while the original task is not obscured. Therefore, AR shows great potential in intra-operative navigation to provide both safety and efficiency.

In this paper, our aim is to demonstrate that AR can serve as a better, more intuitive medium to deliver seamless surgical assistance and a more natural workflow. Our setup is based on depth sensing technology and the Microsoft HoloLens (Microsoft inc.), an optical see-through, self-contained holographic computer. Our previously developed robotic registration system (Liu, Bowyer, Auvinet, \& y Baena, 2017) was used to automatically measure the pose of a proximal femur phantom, according to which AR guidance for the drilling of a pilot hole in the femoral head is delivered via the HoloLens. This enables the surgeon to operate intuitively, without the need for additional physical guides or monitors. For the purposes of this study, we measured the accuracy with which a pilot hole could be drilled into the femoral head using AR navigation on 30 synthetic specimen. Position and orientation errors between the actual and preoperatively planned holes were measured after completion of each experiment. The results show promising accuracy for both position and orientation.

\section{Materials and Methods}

The AR navigation system includes three parts: the registration module (including the depth camera and the robot), the HoloLens, and a tracked surgical drill. Each of them has its own coordinate system, and the establishment of correspondence between is the key to the navigation performance. In order for the HoloLens to generate visual guidance according to registration results, we use an image marker that can be tracked by both the depth camera and the HoloLens as a common frame so that the femur pose can be transformed into HoloLens. Another image marker is attached to the robot base to align the HoloLens with the robot, and the transformation from the marker to the robot is measured before system use. When this marker is recognised by the HoloLens, the correspondence between the robot and the HoloLens is established so that the user can send commands into the robot frame for human-robot interaction.

A cube shaped marker that can be tracked by the HoloLens is fixed on the surgical drill. After calibration between the drill and the marker, the coordinates of the drill can be transformed into the HoloLens frame and compared with the pose of the target femur. If the drill is properly aligned with the pre-operative plan, the HoloLens will inform the user of the results so that the user can proceed with guidance. Figure 1 shows the coordinate relationships between system components, and that a user is performing a drilling procedure with the assistance of AR navigation. 


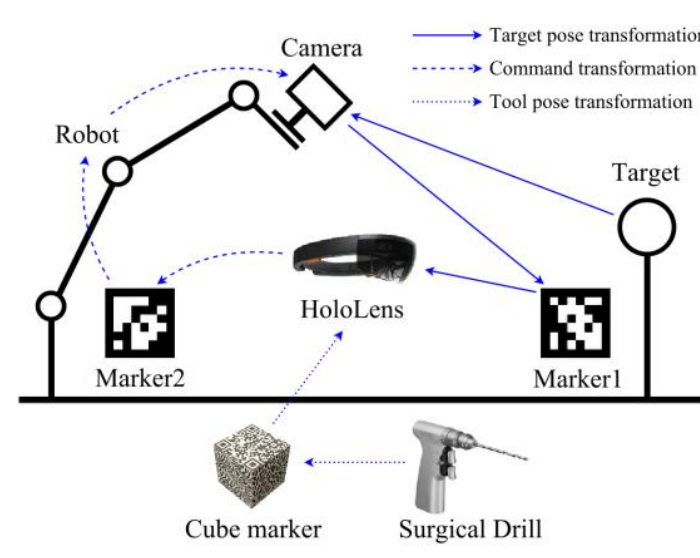

(a) Coordinate relationships between components

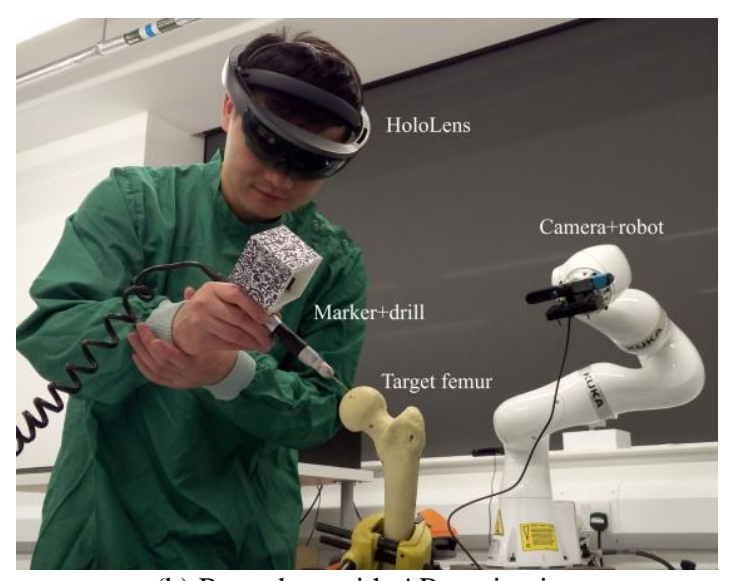

(b) Procedure with AR navigation

Figure 1: Setup and actual use of the AR navigation system.

Experiments were performed to test the accuracy of the proposed AR navigation system. In this paper, we applied the system to the hip resurfacing procedural step in which a guide hole was drilled along the axis of the femoral neck. Conventionally, a specially designed alignment jig is used to find the axis of the femoral neck and to guide drilling. In comparison, AR navigation shows a virtual red arrow to the user, indicating the planned entry point and the drilling direction. If the drill is aligned with the planned drilling path, the whole arrow will turn green so that the user can proceed with drilling. The experiments were performed on foam femur phantoms (Sawbones, Pacific Research Laboratories, Vashon, Washington, U.S.), the surface geometry of which was 3D scanned beforehand for the preoperative plan. Thirty drilling tests were conducted with the assistance of AR navigation, and position and direction errors between the drilled holes and the pre-operative plan were measured after experiments.
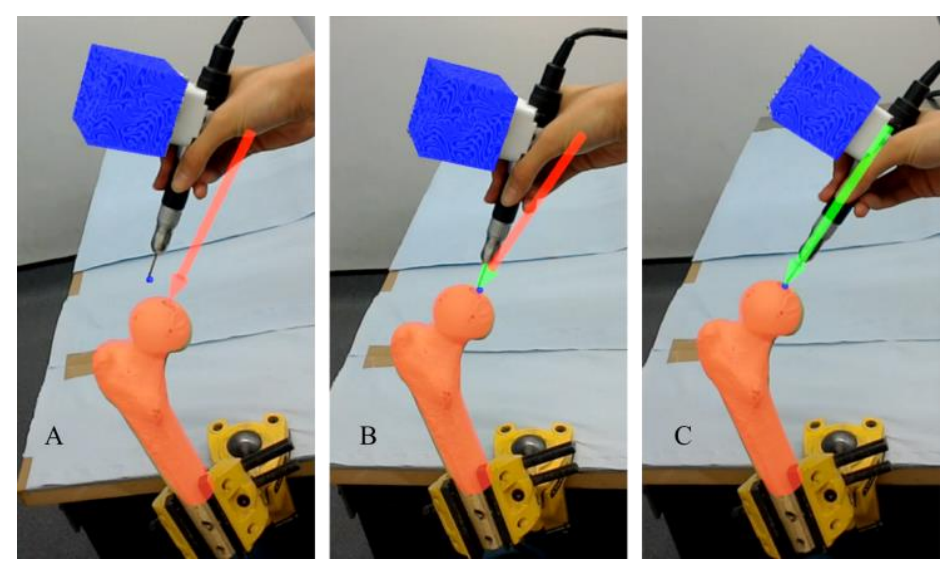

Figure 2: Visual guidance through HoloLens for guide hole drilling. A: Position and direction are inaccurate. B: Position is accurate but direction is inaccurate. C: Position and direction are both accurate.

\section{Results}

Position and direction errors were measured in 3D as absolute values, then direction errors were represented in clinically relevant inclination and version errors. The mean values of absolute position and direction errors are $1.8 \mathrm{~mm}$ (SD: $0.8 \mathrm{~mm}$, range: $0.4 \mathrm{~mm} \sim 3.5 \mathrm{~mm}$ ) and $1.9^{\circ}\left(\mathrm{SD}: 0.9^{\circ}\right.$, range: $0.6^{\circ}$ 
$\sim 3.7^{\circ}$ ), respectively. The direction errors in inclination and version are $-1.6^{\circ}$ (SD: $0.9^{\circ}$, range: $-3.3^{\circ} \sim$ $-0.3^{\circ}$, negative values represent relative varus) and $-0.2^{\circ}$ (SD: $0.8^{\circ}$, range: $-2.2^{\circ} \sim 0.6^{\circ}$, negative values represent retroversion), respectively.

\section{Discussion}

Several studies have been done on the accuracy of guide wire insertion with conventional jigs, patient specific instruments or navigation (Kitada, et al., 2013) (Cobb, et al., 2008) (Olsen, et al., 2010) (Audenaert, et al., 2011). Since the experiment conditions are not the same, the results cannot be compared directly. However, the mean errors of our experiments are comparable to those in their studies, and our standard deviations are generally smaller, indicating satisfactory accuracy and good precision. Additionally, according to the quantitative score table for guide concepts proposed in (Audenaert, et al., 2011), the achieved accuracy is scored as 'good' if position error $\leq 2 \mathrm{~mm}$ and direction error $\leq 2^{\circ}$. Therefore, at least under the somewhat artificial test conditions described here, the accuracy of this AR navigation system is within the 'good' range.

In addition to its accuracy and precision, the AR navigation system also shows promising advantages in terms of surgical workflow. The user can easily interact with the system through some gestures and voice commands, and all the information needed is presented in an intuitive and immersive way. Thus, a user-centered workflow is achieved, where the user can concentrate on the surgical field with fewer distractions. The system's somewhat higher complexity in comparison to traditional navigation is warranted in this proof-of-concept setup, as it is shown to provide a much more informative surgical environment, which can lead to higher efficiency and better surgical accuracy. Significant improvements in commercial AR headsets and low-cost surgical robotic assistants are also expected to alleviate this issue in the future.

\section{References}

Abe, Y., Sato, S., Kato, K., Hyakumachi, T., Yanagibashi, Y., Ito, M., \& Abumi, K. (2013). A novel 3D guidance system using augmented reality for percutaneous vertebroplasty. Journal of Neurosurgery: Spine, 19(4), 492-501.

Audenaert, E., De Smedt, K., Gelaude, F., Clijmans, T., Pattyn, C., \& Geebelen, B. (2011). A custommade guide for femoral component positioning in hip resurfacing arthroplasty: development and validation study. Computer Aided Surgery, 16(6), 304-309.

Cobb, J. P., Kannan, V., Dandachli, W., Iranpour, F., Brust, K. U., \& Hart, A. J. (2008). Learning how to resurface cam-type femoral heads with acceptable accuracy and precision: the role of computed tomography-based navigation. JBJS, 90(Supplement_3), 57-64.

De Haan, R., Campbell, P. A., Su, E. P., \& De Smet, K. A. (2008). Revision of metal-on-metal resurfacing arthroplasty of the hip. Bone \& Joint Journal, 90(9), 1158-1163.

Kitada, M., Sakai, T., Murase, T., Hanada, T., Nakamura, N., \& Sugano, N. (2013). Validation of the femoral component placement during hip resurfacing: a comparison between the conventional jig, patient-specific template, and CT-based navigation. The International Journal of Medical Robotics and Computer Assisted Surgery, 9(2), 223-229.

Liu, H., Bowyer, S., Auvinet, E., \& y Baena, F. R. (2017). A SMART REGISTRATION ASSISTANT FOR JOINT REPLACEMENT: A CONCEPT DEMONSTRATION. Bone Joint J, 99(SUPP 20), 58-58.

Marescaux, J., Diana, M., \& Soler, L. (2013). Augmented reality and minimally invasive surgery. Journal of Gastroenterology and Hepatology Research, 2(5), 555-560. 
Olsen, M., Chiu, M., Gamble, P., Boyle, R. A., Tumia, N., \& Schemitsch, E. H. (2010). A comparison of conventional guidewire alignment jigs with imageless computer navigation in hip resurfacing arthroplasty. JBJS, 92(9), 1834-1841.

Williams, D., Royle, M., \& Norton, M. (2009). Metal-on-metal hip resurfacing: the effect of cup position and component size on range of motion to impingement. The Journal of arthroplasty, 24(1), 144-151. 